\title{
Substituent effects on the formation of benzyl ions from ortho- methoxy substituted 1,1-diarylalkanes under electron ionization: correlations between the abundance of the process and the ${ }^{13} \mathrm{C}-\mathrm{NMR}$ chemical shifts of the neutral precursors
}

\author{
Leopoldo Ceraulo, ${ }^{* a}$ Felice Filizzola, ${ }^{a}$ Gianfranco Fontana, ${ }^{b}$ Liliana Lamartina, ${ }^{a}$ \\ and Maria C. Natoli ${ }^{b}$ \\ ${ }^{a}$ Dipartimento di Chimica e Tecnologie Farmaceutiche, via Archirafi 32, I-90123 Palermo, \\ Italy, and ${ }^{\boldsymbol{b}}$ Dipartimento di Chimica Organica 'E. Paternò', viale Delle Scienze, Parco \\ d'Orleans, I-90128 Palermo, Italy \\ E-mail: 1ceraulo@unipa.it
}

Dedicated to Professor Domenico Spinelli on his $70^{\text {th }}$ birthday

(received 13 Oct 02; accepted 09 Dec 02; published on the web 17 Dec 02)

\begin{abstract}
Good linear relationships have been obtained between the abundance of the formation process of benzyl ions from ortho-methoxy- substituted 1,1-diarylalkanes, through consecutive rearrangement reactions induced by electron ionization, and ${ }^{13} \mathrm{C}$ - NMR chemical shift values of $\mathrm{C}-1$ of the ortho-methoxy- substituted aromatic ring of the neutral precursors for a large number of compounds: 1,1-diphenylethanes 1-19, 2-methyl-1,1-diphenylpropanes 20-37, and 1,1,1trichloro-2,2-diphenylethanes 38-44. This result is satisfying as good linear relationships between the ion abundances in the mass spectrum and the substituent effects are not easily obtained. Further, the abundance of the process depends on the effects of substituents linked to the ortho-methoxy- substituted benzene ring (ring A) while measurable effects of substituents linked to the other benzene ring (ring B) have not been observed.
\end{abstract}

Keywords: 1,1-diarylalkanes, electron ionization, rearrangement processes, substituent effects, ${ }^{13} \mathrm{C}$ NMR Chemical Shifts, cross-correlations

\section{Introduction}

It has been reported ${ }^{1}$ that the electron ionization (EI) mass spectra (MS) of 1,1-diphenylalkanes bearing an ortho-methoxy group are characterized by the presence of abundant benzyl (or tropylium) ions, which are completely absent in derivatives lacking the ortho- methoxy 
substituent. In fact, the EI/MS of such ortho-substituted compounds are dominated by the molecular ion $\left(\mathbf{M}^{+\cdot}\right)$, the diphenylmethyl cation a (arising from loss of the alkyl group linked to the benzyl carbon, as a radical), and the benzyl ion $\mathbf{b}$, as can be seen in the $70 \mathrm{eV} \mathrm{EI/MS}$ of 2methoxy-1,1-diphenylethane (1) (Figure 1).

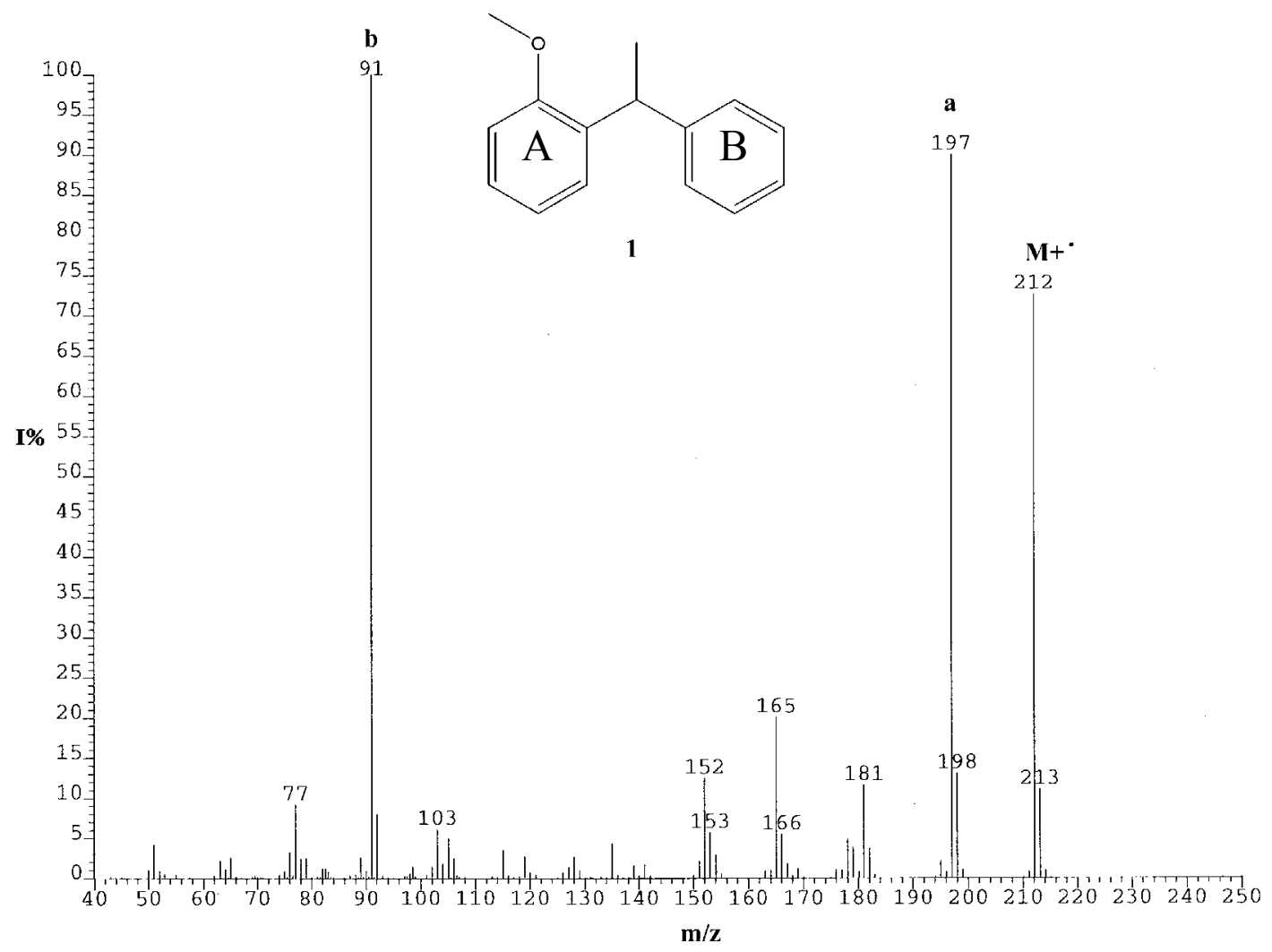

Figure 1. $70 \mathrm{eV}$ EI/MS of 2-methoxy-1,1-diphenylethane (1).

The presence of abundant metastable ions generated in the first field-free region, as well as the presence of the appropriate peaks in mass analyzed ion kinetic energy (MIKE)- or linked scan $\left(\mathrm{B} / \mathrm{E}=\right.$ constant, $\mathrm{B}^{2} / \mathrm{E}=$ constant $)$ spectra, indicates the ion $\mathbf{a}$ as the precursor of the ion $\mathbf{b}^{2,3}$ It has been unequivocally demonstrated by experiments with ${ }^{2} \mathrm{H}$ - and ${ }^{13} \mathrm{C}$-labeled compounds that the whole process of benzyl ion formation involves migration of the methylene residue of the methoxy group linked to the 2-position of an aromatic ring (ring A) to the other aromatic ring (ring B). ${ }^{1-4}$ Further investigation revealed that such a reaction occurs also for ortho-alkoxy derivatives (OEt, OiPr) by migration of an alkylidene residue other than methylene, as well as for ortho-alkyl-hetero (NHMe, $\mathrm{NMe}_{2}, \mathrm{SMe}$ ) 1,1-diarylalkanes. ${ }^{4}$ Finally, it has been determined that the formation of the benzyl- (or tropylium) ions $\mathbf{b}$ constitutes the main unimolecular decomposition reaction of ortho-alkylhetero- substituted diaryl- or alkyldiaryl- or triaryl- methyl cations, a, generated either under electron ionization ${ }^{5}$ or chemical ionization conditions. ${ }^{6}$ 
This previously unreported formation of benzyl ions from diphenylmethane derivatives ${ }^{7}$ through skeletal rearrangement induced by proximity effects is therefore to be considered an important general fragmentation reaction of the cations a occurring over a wide range of internal energy. From a mechanistic point of view it has been suggested ${ }^{1,4}$ that the formation of the ion $\mathbf{b}$ involves consecutive rearrangements (steps 1 and 2) of ortho-methoxy diphenylmethyl cations a, followed by a simple cleavage reaction (step 3) as shown in Scheme 1 for compound $\mathbf{1}$.

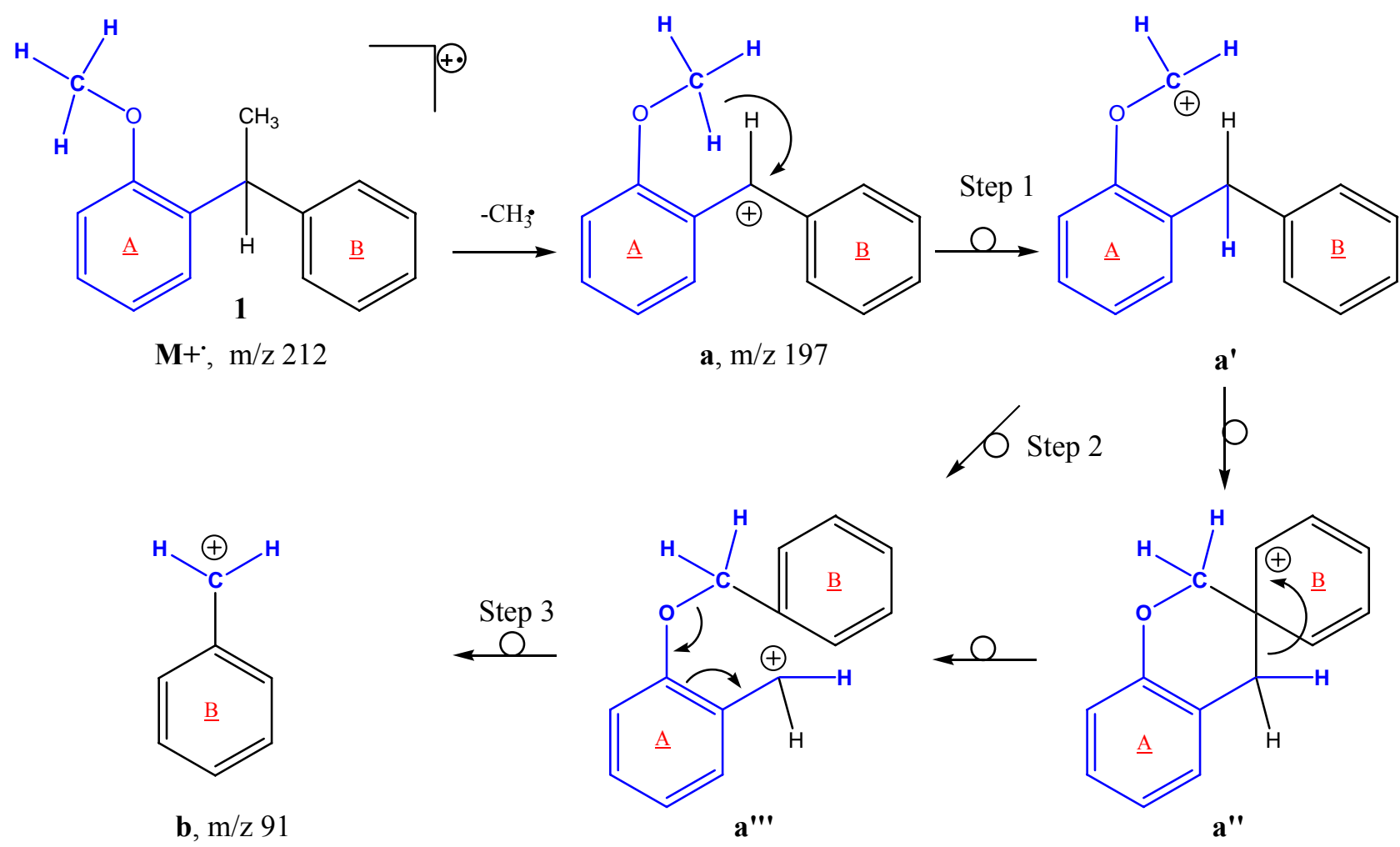

\section{Scheme 1}

In particular, a hydrogen migration as hydride from the $\alpha$-position of the ortho-methoxy group to the carbenium center affords the rearrangement ion a' (Step 1). This latter undergoes a further rearrangement to $\mathbf{a}^{\prime \prime \prime}$ by electrophilic attack of the charged alkylidene (Step 2), probably through a six-membered transition state $\left(\mathbf{a}^{\prime \prime}\right)$, followed by a carbon-carbon displacement reaction. Finally, the benzylic cleavage of ion $\mathbf{a}^{\prime \prime \prime}$ gives the rearrangement benzyl ion $\mathbf{b}$.

The small kinetic energy release values, calculated from the width of the peak at half-height $\left(\mathrm{T}_{1 / 2}\right)$ in the MIKE spectra for the $\mathbf{a} \rightarrow \mathbf{b}$ process, agree with a last step involving a simple cleavage reaction. ${ }^{2}$ The isotopic effect indicates that the hydrogen migration affording the rearrangement ion a' is the rate- determining process, and the close values in $70 \mathrm{eV}-\left(\mathrm{k}_{\mathrm{H}} / \mathrm{k}_{\mathrm{D}}=\right.$ 1.4) ${ }^{1,3}$ and in MIKE- $\left(\mathrm{k}_{\mathrm{H}} / \mathrm{k}_{\mathrm{D}}=1.6\right),{ }^{2}$ spectra agree with a loose transition state for the hydrogen migration. ${ }^{8}$ 
An appropriate geometry of the transition state that should account for the hydride migration involves the ring A being perpendicular to the $\mathrm{sp}^{2}$ system of the charged benzyl carbon, with the methyl of the ortho-alkylhetero group oriented toward the carbenium center. ${ }^{2}$

In order to achieve detailed mechanistic insight and information on the arrangement of the transition state related to the process involving the hydrogen migration, the determination of substituent effects on the abundance of the benzyl ion formation process could be used as a valuable tool. Hence, in this work we correlate the substituent effects with the values of the ratio $\mathbf{Z}$ between the abundance of the benzyl ion $\mathbf{b}$ and the sum of the abundances of the benzyl ion $\mathbf{b}$ with that of its precursor ion $\mathbf{a}\left[\mathbf{Z}=\mathrm{I}_{\mathrm{b}} \% /\left(\mathrm{I}_{\mathrm{a}} \%+\mathrm{I}_{\mathrm{b}} \%\right)\right]$. The ratio $\mathbf{Z}$ is, in good approximation, $\mathrm{a}$ measure of the fraction of ions a reacting to form ions $\mathbf{b}$. It has been proved previously that $\mathbf{Z}$ is a valuable quantitative parameter for correlating the abundance of the process with the degreeof-freedom effect, as well as with the approximate activation energy. ${ }^{2}$

\section{Results and Discussion}

As our starting point, we compared the $\mathbf{Z \%}$ value of the 2-methoxy-1,1-diphenylethane (1) with those of derivatives $\mathbf{2 - 1 1}$ characterized by an unsubstituted ring B and bearing substituents at the 4- and/or 5-position in the ring A (Table 1).

The EI/MS (see Experimental Section) of the compounds 1-11 are dominated by the peaks corresponding to the molecular ion $\mathbf{M}^{+\bullet}$, the ion $\mathbf{a}$, and the rearrangement benzyl ion, $\mathbf{b}(\mathrm{m} / \mathrm{z}$ 91).

Analysis of the 5-substituted derivatives 2-5 shows a $\mathbf{Z \%}=57$ for the 5-methyl-derivative $\mathbf{2}$, very close to that of $\mathbf{1}(\mathbf{Z} \%=53)$, which agrees with the poor electronic effects of the methyl group. A significant small increase of $\mathbf{Z \%}$ with respect to $\mathbf{1}$ is observed for the 5-methoxy derivative $3(\mathbf{Z \%}=63)$, the 5-nitro derivative $4(\mathbf{Z \%}=64)$ and the 5-bromo derivative $5(\mathbf{Z \%}=$ 62). These substituents linked at the 5-position exert a small electron-withdrawing effect with respect to the meta- positions (C-1 and C-3).

Similarly, the poor effect of a methyl group linked to the 4-position is evidenced by the value of $\mathbf{Z \%}=55$ for the 4-methyl derivative $\mathbf{6}$. On the contrary, a relevant reduction in $\mathbf{Z \%}$ is achieved by the presence of strong para- electron-donating groups linked at the 4-position of the ring $\mathrm{A}$, as observed for the 4-methoxy derivative $7(\mathbf{Z \%}=34)$, the 4-amino-5-methoxy derivative $8(\mathbf{Z \%}=20)$, the 4- $N$-methylamino-5-methoxy derivative $9(\mathbf{Z \%}=11)$, and the 4$N, N$-dimethylamino-5-methoxy derivative $\mathbf{1 0}(\mathbf{Z \%}=17)$. Finally, the biggest $\mathrm{Z} \%$ value is observed for 5-methoxy-4-nitro-1,1-diphenylethane (11) $(\mathbf{Z \%}=89)$.

All these findings revealed a meaningful dependence of $\mathbf{Z} \%$ on the substituents of the ring A. In particular, the substituents which exert an electron-withdrawing effect on C-1 increase the abundance of the process, while strong electron-donating groups produce a dramatic decrease in $\mathbf{Z} \%$. In fact, the lower is the electron density on $\mathrm{C}-1$ of the ring $\mathrm{A}$ of the ion a is, the higher is the fraction of ions a affording ions $\mathbf{b}$. 
In addition to such a qualitative approach, it seemed of interest to verify whether linear relationships occur with the electronic effects of the substituents. However, the use of electronic constants in our substrates, which are characterized by the presence of several substituents, could be strongly affected by severe limitations owing to the occurrence of ortho- interactions, which are not easily evaluated, a priori.

The ${ }^{13} \mathrm{C}$ NMR chemical shift constitutes a physical parameter related to the electronic density of the carbon atoms. ${ }^{9}$ However, NMR experiments on the diaryl cations a are complicated by the difficulty of generating them in the solution phase. In fact, this should involve the use of superacids on the appropriate precursors, and low temperature experiments. ${ }^{10}$ Hence, the reasonable assumption that a substituent could play a role in the same direction either in ions a or in their neutral precursors prompted us to correlate the ${ }^{13} \mathrm{C}$ NMR chemical shift values (used as electronic parameters) of the neutral precursors, the 2-methoxy-1,1-diphenylethanes, with $\mathbf{Z \%}$. This approach has been applied successfully in the study of the reactivity of carbanions in solution. $^{11}$

Then, taking the chemical shift value of $\mathrm{C}-1$ carbon atom of the ring A for $\mathbf{1}$ as reference, a correlation between the substituent- induced chemical shift variations (cs) for compounds 1-11 (Table 1) and the $\mathbf{Z \%}$ values was carried out.

The results shown in Figure 3 show that a good linear correlation (s $3.67 \pm 0.21$, i $57.59 \pm$ $1.40, n 11, r 0.986)$ has been obtained.

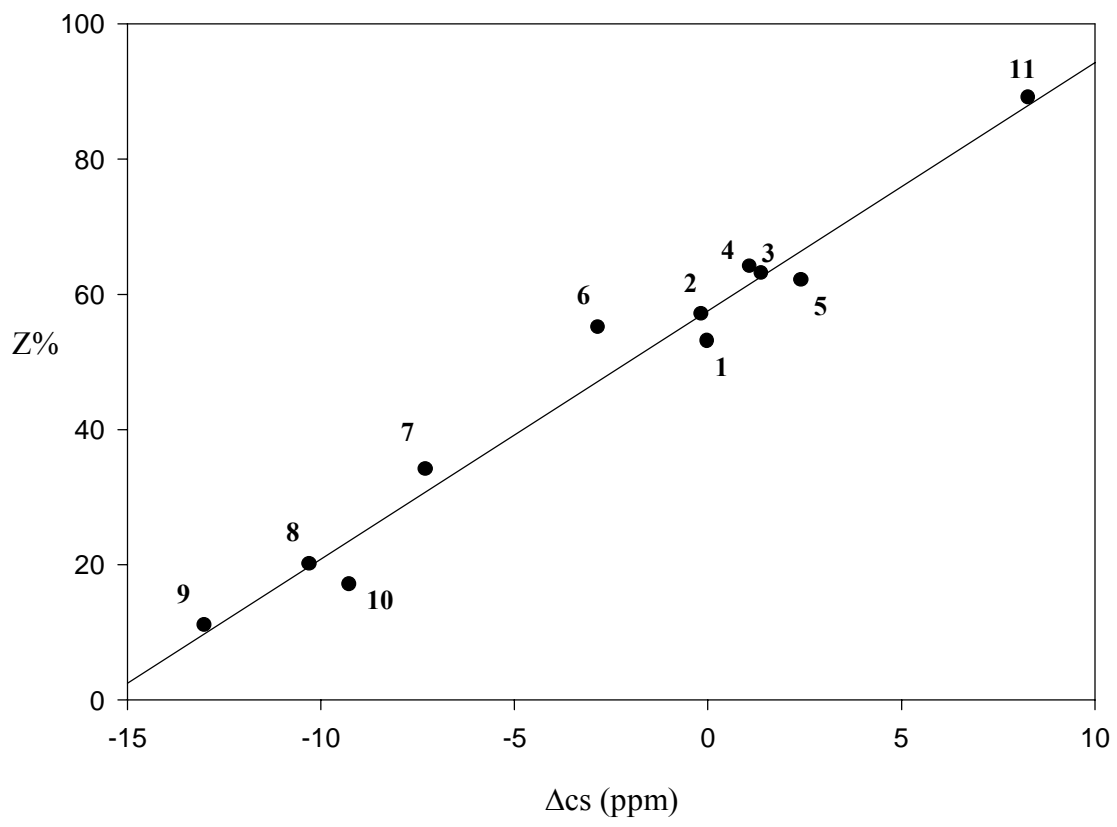

Figure 3. Plot of Z\% values vs. cs (C-1) of compounds 1-11. Refer to Table 1 for the values. 
Once the close dependence of the process on the electronic density of C-1 has been asserted, it seemed of interest to verify a possible influence of substitutions at the ring B. Comparison of the $\mathbf{Z \%}$ value of the 4'-methoxy derivative $\mathbf{1 2}$ (Table 1) with that of $\mathbf{1}$ shows that the presence of the 4 '-methoxy group in the ring B does not produce significant change $(\mathbf{Z} \%=57)$. Similarly, the introduction of the 4'-nitro group into the structure 11, i.e., compound 13, leads to a value of $\mathbf{Z} \%=87$, practically identical to the $\mathbf{Z} \%$ value of $\mathbf{1 1}$. Incidentally, the $70 \mathrm{eV} \mathrm{EI} / \mathrm{MS}$ of the dimethoxy- ${ }^{2} \mathrm{H}_{6}$ isotopomer, 13-d $\mathbf{d}_{\mathbf{6}}$, gives evidence of an isotopic effect, calculated by the ratio of $\mathrm{I} \%$ of $m / z 136$ (ion $\mathbf{b}$ for $\mathbf{1 3}$ ) $/ \mathrm{I} \%$ of $m / z 138$ (ion $\mathbf{b}$ for $\mathbf{1 3}-\mathbf{d}_{\mathbf{6}}$ ), $\mathrm{K}_{\mathrm{H}} / \mathrm{K}_{\mathrm{D}}=1.5$, very close to that previously determined for other derivatives ${ }^{1,3}$ lacking electron-withdrawing substituents on the ring $\mathrm{B}$. This means that the hydride migration step 1 (Scheme 1) constitutes the rate-determining reaction also in the presence of a para-nitro group in the ring $\mathrm{B}$, which should enhance the activation energy of the electrophilic substitution involved in Step 2.

Furthermore, good linear relationships between $\mathbf{Z \%}$ and $\mathbf{c s}$ of $\mathrm{C}-1$ for all 1-11, together with the polysubstituted compounds 12-19, have been obtained (s $3.74 \pm 0.25$, i $60.84 \pm 1.50, n 19, r$ 0.963). The substantial absence of effects of the substituents linked to the ring $B$ on the abundance of the process was also stressed by the lack of any correlation between $\mathbf{Z \%}$ and the ${ }^{13} \mathrm{C}$-NMR chemical shift values of $\mathrm{C}-1$ ' of the ring $\mathrm{B}$.

Finally in this context, we extended our investigation to substrates with complex substitution patterns, namely the ortho-methoxy 2-methyl-1,1-diphenylpropanes 20-37 (Table 2) and orthomethoxy-1,1,1-trichloro-2,2-diphenylethanes, 38-44 (Table 3), which generate ions a by loss of isopropyl and trichloromethyl radicals, respectively, from the molecular ion. In order to calculate the $\Delta \mathbf{c s}$ values of $\mathrm{C}-1$, the compounds $\mathbf{2 0}$ and $\mathbf{3 8}$ have been used as references for the $\mathbf{2 0 - 3 7}$ and 38-44 series, respectively.

Satisfactory linear relationships have also been obtained for both series, 20-37 (s $2.33 \pm 0.20$, i $53.14 \pm 1.05, n 18, r$ 0.947), and 38-44 (s $2.87 \pm 0.49, i 49.78 \pm 2.31, n$ 7, $r$ 0.935). 
Table 1. ${ }^{13} \mathrm{C}$ - NMR data ${ }^{a}$ and $\mathrm{Z} \%{ }^{b}$ of $\mathbf{1 - 1 9}$

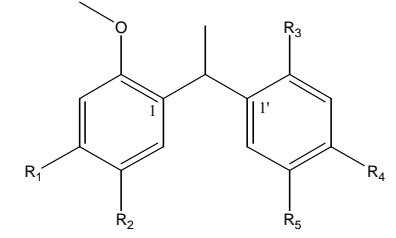

\begin{tabular}{|c|c|c|c|c|c|c|c|c|c|}
\hline Compd. & $\mathrm{R}_{1}$ & $\mathrm{R}_{2}$ & $\mathrm{R}_{3}$ & $\mathrm{R}_{4}$ & $\mathrm{R}_{5}$ & C-1 & C-1' & $\begin{array}{c}\mathrm{cs} \\
(\mathrm{C}-1)\end{array}$ & $Z \%$ \\
\hline 1 & $\mathrm{H}$ & $\mathrm{H}$ & $\mathrm{H}$ & $\mathrm{H}$ & $\mathrm{H}$ & 134.76 & 146.28 & 0.00 & 53 \\
\hline 2 & $\mathrm{H}$ & $\mathrm{Me}$ & $\mathrm{H}$ & $\mathrm{H}$ & $\mathrm{H}$ & 134.61 & 146.41 & -0.15 & 57 \\
\hline 3 & $\mathrm{H}$ & $\mathrm{OMe}$ & $\mathrm{H}$ & $\mathrm{H}$ & $\mathrm{H}$ & 136.16 & 145.97 & 1.40 & 63 \\
\hline 4 & $\mathrm{H}$ & $\mathrm{NO}_{2}$ & $\mathrm{H}$ & $\mathrm{H}$ & $\mathrm{H}$ & 135.86 & 144.63 & 1.10 & 64 \\
\hline 5 & $\mathrm{H}$ & $\mathrm{Br}$ & $\mathrm{H}$ & $\mathrm{H}$ & $\mathrm{H}$ & 137.19 & 145.44 & 2.43 & 62 \\
\hline 6 & $\mathrm{Me}$ & $\mathrm{H}$ & $\mathrm{H}$ & $\mathrm{H}$ & $\mathrm{H}$ & 131.94 & 146.57 & -2.82 & 55 \\
\hline 7 & $\mathrm{OMe}$ & $\mathrm{H}$ & $\mathrm{H}$ & $\mathrm{H}$ & $\mathrm{H}$ & 127.48 & 146.72 & -7.28 & 34 \\
\hline 8 & $\mathrm{NH}_{2}$ & $\mathrm{OMe}$ & $\mathrm{H}$ & $\mathrm{H}$ & $\mathrm{H}$ & 124.48 & 146.97 & -10.28 & 20 \\
\hline 9 & $\mathrm{NHMe}$ & $\mathrm{OMe}$ & $\mathrm{H}$ & $\mathrm{H}$ & $\mathrm{H}$ & 121.77 & 147.13 & -12.99 & 11 \\
\hline 10 & $\mathrm{NMe}_{2}$ & $\mathrm{OMe}$ & $\mathrm{H}$ & $\mathrm{H}$ & $\mathrm{H}$ & 125.51 & 146.49 & -9.25 & 17 \\
\hline 11 & $\mathrm{NO}_{2}$ & $\mathrm{OMe}$ & $\mathrm{H}$ & $\mathrm{H}$ & $\mathrm{H}$ & 143.06 & 144.27 & 8.30 & 89 \\
\hline 12 & $\mathrm{H}$ & $\mathrm{H}$ & $\mathrm{H}$ & $\mathrm{OMe}$ & $\mathrm{H}$ & 135.22 & 138.45 & 0.46 & 57 \\
\hline 13 & $\mathrm{NO}_{2}$ & $\mathrm{OMe}$ & $\mathrm{H}$ & $\mathrm{NO}_{2}$ & $\mathrm{H}$ & 140.47 & 152.25 & 5.71 & 87 \\
\hline 14 & $\mathrm{H}$ & $\mathrm{H}$ & $\mathrm{Cl}$ & $\mathrm{OMe}$ & $\mathrm{H}$ & 133.90 & 135.79 & -0.86 & 66 \\
\hline 15 & $\mathrm{H}$ & $\mathrm{H}$ & $\mathrm{Br}$ & $\mathrm{OMe}$ & $\mathrm{H}$ & 133.92 & 137.45 & -0.84 & 68 \\
\hline 16 & $\mathrm{H}$ & $\mathrm{H}$ & $\mathrm{OMe}$ & $\mathrm{H}$ & $\mathrm{H}$ & 134.84 & 134.84 & 0.08 & 67 \\
\hline 17 & $\mathrm{OMe}$ & $\mathrm{H}$ & $\mathrm{H}$ & $\mathrm{OMe}$ & $\mathrm{H}$ & 128.02 & 138.84 & -6.74 & 38 \\
\hline 18 & $\mathrm{OMe}$ & $\mathrm{H}$ & $\mathrm{OMe}$ & $\mathrm{OMe}$ & $\mathrm{H}$ & 127.49 & 127.49 & -7.27 & 42 \\
\hline 19 & $\mathrm{OMe}$ & $\mathrm{Br}$ & $\mathrm{OMe}$ & $\mathrm{OMe}$ & $\mathrm{Br}$ & 128.53 & 128.53 & -6.23 & 37 \\
\hline
\end{tabular}

${ }^{a}$ Chemical shift values for $\mathrm{C}-1$ are in ppm; $\Delta \operatorname{cs}(\mathrm{C}-1)$ values are calculated as differences between $\delta(\mathrm{C}-1)$ of each compound and $\delta(\mathrm{C}-1)$ of compound $1 .{ }^{\mathrm{b}} \mathrm{Z} \%$ are calculated as $\mathrm{I}_{\mathrm{b}} \% /\left(\mathrm{I}_{\mathrm{a}} \%+\mathrm{I}_{\mathrm{b}} \%\right)$ per cent. 
Table 2. ${ }^{13} \mathrm{C}$ NMR data ${ }^{a}$ and $Z \%{ }^{b}$ of $\mathbf{2 0 - 3 7}$

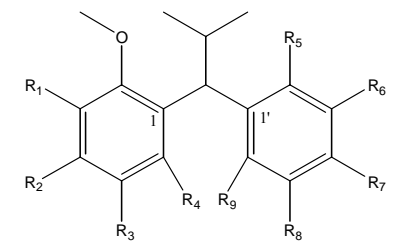

\begin{tabular}{|c|c|c|c|c|c|c|c|c|c|c|c|c|}
\hline Comp. & $\mathrm{R}_{1}$ & $\mathrm{R}_{2}$ & $\mathrm{R}_{3}$ & $\mathrm{R}_{4}$ & $\mathrm{R}_{5}$ & $\mathrm{R}_{6}$ & $\mathrm{R}_{7}$ & $\mathrm{R}_{8}$ & $\mathrm{R}_{9}$ & C-1 & $\begin{array}{c}\mathrm{cs} \\
(\mathrm{C}-1)\end{array}$ & $Z \%$ \\
\hline 20 & $\mathrm{H}$ & $\mathrm{H}$ & $\mathrm{H}$ & $\mathrm{H}$ & $\mathrm{H}$ & $\mathrm{OMe}$ & $\mathrm{OMe}$ & $\mathrm{H}$ & $\mathrm{H}$ & 133.42 & 0.00 & 56 \\
\hline 21 & $\mathrm{H}$ & $\mathrm{OMe}$ & $\mathrm{H}$ & $\mathrm{H}$ & $\mathrm{OMe}$ & $\mathrm{H}$ & $\mathrm{OMe}$ & $\mathrm{H}$ & $\mathrm{H}$ & 126.00 & -7.42 & 36 \\
\hline 22 & $\mathrm{H}$ & $\mathrm{OMe}$ & $\mathrm{H}$ & $\mathrm{H}$ & $\mathrm{H}$ & $\mathrm{OMe}$ & $\mathrm{OMe}$ & $\mathrm{H}$ & $\mathrm{H}$ & 126.00 & -7.42 & 35 \\
\hline 23 & $\mathrm{H}$ & $\mathrm{H}$ & OMe & $\mathrm{H}$ & $\mathrm{OMe}$ & $\mathrm{H}$ & $\mathrm{H}$ & $\mathrm{OMe}$ & $\mathrm{H}$ & 134.58 & 1.16 & 60 \\
\hline 24 & $\mathrm{H}$ & $\mathrm{H}$ & OMe & $\mathrm{H}$ & $\mathrm{H}$ & OMe & $\mathrm{OMe}$ & $\mathrm{H}$ & $\mathrm{H}$ & 135.02 & 1.60 & 49 \\
\hline 25 & $\mathrm{OMe}$ & $\mathrm{H}$ & $\mathrm{H}$ & $\mathrm{H}$ & $\mathrm{H}$ & $\mathrm{OMe}$ & OMe & $\mathrm{H}$ & $\mathrm{H}$ & 138.95 & 5.53 & 66 \\
\hline 26 & $\mathrm{OMe}$ & $\mathrm{OMe}$ & $\mathrm{H}$ & $\mathrm{H}$ & $\mathrm{H}$ & $\mathrm{OMe}$ & $\mathrm{OMe}$ & $\mathrm{H}$ & $\mathrm{H}$ & 131.28 & -2.14 & 43 \\
\hline 27 & $\mathrm{H}$ & $\mathrm{OMe}$ & OMe & $\mathrm{H}$ & $\mathrm{H}$ & OMe & $\mathrm{OMe}$ & $\mathrm{H}$ & $\mathrm{H}$ & 125.00 & -8.42 & 35 \\
\hline 28 & $\mathrm{H}$ & $\mathrm{H}$ & $\mathrm{Me}$ & $\mathrm{H}$ & $\mathrm{OMe}$ & $\mathrm{H}$ & $\mathrm{H}$ & $\mathrm{Me}$ & $\mathrm{H}$ & 133.11 & -0.31 & 58 \\
\hline 29 & $\mathrm{H}$ & $\mathrm{H}$ & $\mathrm{Me}$ & $\mathrm{H}$ & $\mathrm{H}$ & $\mathrm{OMe}$ & $\mathrm{OMe}$ & $\mathrm{H}$ & $\mathrm{H}$ & 133.14 & -0.28 & 45 \\
\hline 30 & $\mathrm{H}$ & $\mathrm{Br}$ & $\mathrm{OMe}$ & $\mathrm{H}$ & $\mathrm{OMe}$ & $\mathrm{H}$ & $\mathrm{Br}$ & $\mathrm{OMe}$ & $\mathrm{H}$ & 132.75 & -0.70 & 47 \\
\hline 31 & $\mathrm{H}$ & $\mathrm{OMe}$ & $\mathrm{Br}$ & $\mathrm{H}$ & $\mathrm{OMe}$ & $\mathrm{H}$ & $\mathrm{OMe}$ & $\mathrm{Br}$ & $\mathrm{H}$ & 126.65 & -6.77 & 36 \\
\hline 32 & $\mathrm{Br}$ & $\mathrm{Br}$ & OMe & $\mathrm{H}$ & $\mathrm{OMe}$ & $\mathrm{Br}$ & $\mathrm{Br}$ & OMe & $\mathrm{H}$ & 137.03 & 3.61 & 60 \\
\hline 33 & $\mathrm{Br}$ & OMe & $\mathrm{Br}$ & $\mathrm{H}$ & $\mathrm{H}$ & $\mathrm{OMe}$ & OMe & $\mathrm{Br}$ & $\mathrm{Br}$ & 135.36 & 1.94 & 60 \\
\hline 34 & $\mathrm{OMe}$ & $\mathrm{H}$ & $\mathrm{Br}$ & $\mathrm{Br}$ & $\mathrm{H}$ & $\mathrm{OMe}$ & $\mathrm{OMe}$ & $\mathrm{Br}$ & $\mathrm{Br}$ & 137.20 & 3.78 & 63 \\
\hline 35 & $\mathrm{H}$ & $\mathrm{OMe}$ & $\mathrm{H}$ & $\mathrm{Cl}$ & $\mathrm{H}$ & OMe & $\mathrm{OMe}$ & $\mathrm{H}$ & $\mathrm{H}$ & 123.96 & -9.46 & 34 \\
\hline 36 & $\mathrm{H}$ & $\mathrm{NO}_{2}$ & OMe & $\mathrm{H}$ & $\mathrm{OMe}$ & $\mathrm{H}$ & $\mathrm{NO}_{2}$ & OMe & $\mathrm{H}$ & 138.81 & 5.39 & 72 \\
\hline 37 & $\mathrm{H}$ & $\mathrm{OMe}$ & $\mathrm{NO}_{2}$ & $\mathrm{H}$ & $\mathrm{OMe}$ & $\mathrm{H}$ & OMe & $\mathrm{NO}_{2}$ & $\mathrm{H}$ & 123.96 & -9.46 & 33 \\
\hline
\end{tabular}

${ }^{a}$ Chemical shift values for $\mathrm{C}-1$ are in ppm; $\Delta \mathrm{cs}(\mathrm{C}-1)$ values are calculated as differences between $\delta(\mathrm{C}-1)$ of each compound and $\delta(\mathrm{C}-1)$ of compound $20 .{ }^{\mathrm{b}} \mathrm{Z} \%$ are calculated as $\mathrm{I}_{\mathrm{b}} \% /\left(\mathrm{I}_{\mathrm{a}} \%+\mathrm{I}_{\mathrm{b}} \%\right)$ per cent.

\section{Conclusions}

The choice of the ${ }^{13} \mathrm{C}$-NMR chemical shift values of C-1 of the ring A of the neutral precursor of the benzyl ions $\mathbf{b}$, formed by consecutive rearrangements of the diarylmethyl cations $\mathbf{a}$, leads to good linear relationships with the fraction of ions $\mathbf{a}$ affording the ions $\mathbf{b}$, measured by the ratio $\mathbf{Z} \%$, for a large number of compounds.

This result is quite satisfying, as good linear relationships between the ion abundances in the mass spectrum and the substituent constants are not easily obtained. In fact, this approach does 
suffer severe limitations owing to: (i) the fact that the ion abundances in the mass spectrum results from competitive and consecutive reactions of precursors with a relatively large range of internal energy; (ii) there is the possible occurrence of isolated electronic states, not easily predictable a priori and, (iii), there is the degree-of-freedom effect on the fragment-ion abundances. $^{8}$

Further, our findings give evidence that the abundance of the formation process of benzyl ions $\mathbf{b}$ depends on the effects of substituents linked to the ortho-methoxy- substituted benzene ring (ring A). In particular, the lower is the electron density of $\mathrm{C}-1$ of the ring $\mathrm{A}$ of the neutral precursor, the higher is the fraction of ion a which give benzylic ion $\mathbf{b}$. On the other hand, measurable effects of substituents linked to the other benzene ring (ring B) have not been observed.

Finally, it seems possible to estimate the ion-abundances in the EI/MS of ortho-alkylheterosubstituted 1,1-diarylalkanes by means of a unique parameter which reflects the overall effects of the substituents, that is, the $\mathrm{C}-1{ }^{13} \mathrm{C}$ NMR value of the neutral precursor.

Table 3. ${ }^{13} \mathrm{C}$ NMR data ${ }^{a}$ and $\mathrm{Z} \%{ }^{b}$ of $38-44$

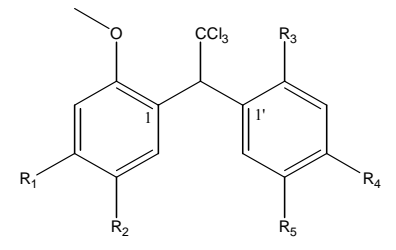

\begin{tabular}{|c|c|c|c|c|c|c|c|c|}
\hline Comp. & $\mathrm{R}_{1}$ & $\mathrm{R}_{2}$ & $\mathrm{R}_{3}$ & $\mathrm{R}_{4}$ & $\mathrm{R}_{5}$ & C-1 & $\operatorname{cs}(\mathrm{C}-1)$ & $\mathrm{Z} \%$ \\
\hline 38 & $\mathrm{H}$ & $\mathrm{H}$ & $\mathrm{H}$ & $\mathrm{OMe}$ & $\mathrm{H}$ & 127.76 & 0.00 & 56 \\
\hline 39 & $\mathrm{H}$ & $\mathrm{OMe}$ & $\mathrm{OMe}$ & $\mathrm{H}$ & $\mathrm{OMe}$ & 126.33 & 0.57 & 56 \\
\hline 40 & $\mathrm{OMe}$ & $\mathrm{H}$ & $\mathrm{OMe}$ & $\mathrm{OMe}$ & $\mathrm{H}$ & 120.36 & -7.40 & 29 \\
\hline 41 & $\mathrm{H}$ & $\mathrm{Me}$ & $\mathrm{OMe}$ & $\mathrm{H}$ & $\mathrm{Me}$ & 127.22 & -0.54 & 52 \\
\hline 42 & $\mathrm{OMe}$ & $\mathrm{OMe}$ & $\mathrm{OMe}$ & $\mathrm{OMe}$ & $\mathrm{OMe}$ & 118.97 & -8.79 & 22 \\
\hline 43 & $\mathrm{Br}$ & $\mathrm{OMe}$ & $\mathrm{OMe}$ & $\mathrm{Br}$ & $\mathrm{OMe}$ & 126.81 & -0.95 & 40 \\
\hline 44 & $\mathrm{NO}_{2}$ & $\mathrm{OMe}$ & $\mathrm{OMe}$ & $\mathrm{NO}_{2}$ & $\mathrm{OMe}$ & 132.53 & 4.77 & 58 \\
\hline
\end{tabular}

a Chemical shift values for $\mathrm{C}-1$ and $\mathrm{C}-1$ ' are in $\mathrm{ppm} ; \Delta \operatorname{cs}(\mathrm{C}-1)$ values are calculated as differences between $\delta(\mathrm{C}-1)$ of each compound and $\delta(\mathrm{C}-1)$ of compound $38{ }^{\mathrm{b}} \mathrm{Z} \%$ are calculated as $\mathrm{I}_{\mathrm{b}} \% /\left(\mathrm{I}_{\mathrm{a}} \%+\mathrm{I}_{\mathrm{b}} \%\right)$ per cent. 


\section{Experimental Section}

General Procedures. Melting points were measured on a Büchi 510 melting point apparatus and are uncorrected. ${ }^{1} \mathrm{H}$ - and ${ }^{13} \mathrm{C}$ - NMR spectra were recorded on a Bruker AC 250 spectrometer in deuteriochloroform solutions. ${ }^{13} \mathrm{C}$ - NMR chemical shifts (in ppm) are given from $\mathrm{CDCl}_{3}(77.00)$ and were taken from fully decoupled spectra. The carbon signals were assigned on the basis of known substituent effects and, when necessary, multiplicities were also determined by proton gated decoupled experiments. For compounds 1-15, 17, 20, 22, 24-27, 29, 33, 34, 35 and 38 the $\mathrm{C}^{\prime}$ symbol refers to carbon atoms of ring $\mathrm{B}$.

Low resolution (LR) - and high resolution (HR) - mass spectra were recorded by the Autospec Ultima o-TOF (Micromass, U.K.) mass spectrometer connected with a GC system HP 6890 series (Hewlett Packard) of the "Rete di Spettrometria di Massa-CNR" operating in the Dipartimento di Chimica e Tecnologie Farmaceutiche-Palermo. LRMS were performed under the following experimental conditions: electron beam energy, $70 \mathrm{eV}$; source temperature $220{ }^{\circ} \mathrm{C}$; trap current $250 \mu \mathrm{A}$; accelerating voltage $8 \mathrm{kV}$; resolution power 1,500. Even though the analyzed samples 1-44 were pure by TLC, in order to avoid possible interference peaks, they were introduced into the ion source through the GC system. The GC conditions were: injector temperature $250{ }^{\circ} \mathrm{C}$; column $\mathrm{AT}^{\mathrm{TM}-5}$ (from Alltech), film thickness $0.25 \mu \mathrm{m}$, length $30 \mathrm{~m}$, ID $0.25 \mathrm{~mm}$, carrier gas (helium) flow $1.0 \mathrm{~mL} / \mathrm{min}$, Ramp $100{ }^{\circ} \mathrm{C}(3 \mathrm{~min}), 100-300{ }^{\circ} \mathrm{C}\left(10{ }^{\circ} \mathrm{C} / \mathrm{min}\right)$, $300{ }^{\circ} \mathrm{C}(7 \mathrm{~min})$. Better reproducibility of the ion intensities was achieved by averaging the spectra from the initial half-height to the end half-height of the GC peak (3-5 scans averaged for each analysis) with background subtraction. The values of $\mathbf{Z \%}$ were reproducible $( \pm 2)$. The $\mathbf{Z \%}$ values reported in Tables 1-3 and the ion abundances (with respect to the base peak) of the partial mass spectra $\left(\mathbf{M}^{+\bullet}\right.$, ion $\mathbf{a}$, ion $\mathbf{b}$, which are responsible of the most abundant peaks in the MS) of compounds 1-44 refer to the mean of three independent analyses for each sample.

The elemental composition of compounds has been confirmed by HRMS measurements, with resolving power of 5,000 (10\% resolution valley definition) using PFK as internal standard. All the values found were within \pm 10 ppm of the calculated ones.

IR spectra were obtained with a Perkin-Elmer Model 1310 IR spectrophotometer.

All commercial products were from Aldrich. Silica gel 60 (Merck, 0.06-0.2 mm) was used for column chromatography. Analytical- and ${ }^{1} \mathrm{H}-\mathrm{NMR}$ data confirmed the assigned structures for all synthesized compounds.

Compounds $1,{ }^{3} \mathbf{1 8}, \mathbf{1 9}, \mathbf{3 0}-\mathbf{3 3},{ }^{12} \mathbf{2 0}-\mathbf{2 5}, \mathbf{2 7},{ }^{1} \mathbf{3 6}, \mathbf{3 7}{ }^{13}$ were synthesized according to reported methods. For these compounds we report the ${ }^{13} \mathrm{C}$ NMR chemical shift values, not previously reported. Syntheses and ${ }^{13} \mathrm{C}$ NMR chemical shift values for compounds $\mathbf{3 8} \mathbf{- 4 2}$ have been reported previously. ${ }^{11}$ For these compounds we report only the partial mass spectra $\left(\mathbf{M}^{+\bullet}\right.$, ion $\mathbf{a}$, and ion $\mathbf{b})$. 
1-(2-Methoxyphenyl)-1-phenylethane (1). ${ }^{13} \mathrm{C}$ NMR: $20.83\left(\mathrm{CHCH}_{3}\right), 37.33\left(\mathrm{CHCH}_{3}\right), 55.19$ $\left(\mathrm{OCH}_{3}\right), 110.45$ (C-3), 120.42 (C-5), 125.59 (C-4'), 126.97 (C-4), 127.55 (C-6), 127.62 (C-2',6'), 128.00 (C-3',5'), 134.76 (C-1), 146.28 (C-1'), 156.71 (C-2). EI/MS, m/z (I\%): 212 (72.78) $\mathbf{M}^{+\bullet}$; 197 (90.10) a; 91 (100.00) b.

1,1-Bis(2,4-dimethoxyphenyl)-ethane (18). ${ }^{13} \mathrm{C} \mathrm{NMR:} 20.25\left(\mathrm{CHCH}_{3}\right), 30.32\left(\mathrm{CHCH}_{3}\right), 55.02$ $\left(\mathrm{OCH}_{3}\right), 55.33\left(\mathrm{OCH}_{3}\right), 98.61$ (2 C-3), 103.60 (2 C-5), 127.49 (2 C-1), 127.92 (2 C-6), 157.88 (2 C-2), 158.92 (2 C-4). EI/MS, m/z (I\%): 302 (37.25) $\mathbf{M}^{+\bullet} ; 287$ (100.00) a; 151 (72.17) b.

1,1-Bis(5-bromo-2,4-dimethoxyphenyl)-ethane (19). ${ }^{13} \mathrm{C} \quad \mathrm{NMR}: 19.81 \quad\left(\mathrm{CHCH}_{3}\right), 30.95$ $\left(\mathrm{CHCH}_{3}\right), 55.99(\mathrm{OCH} 3), 56.47\left(\mathrm{OCH}_{3}\right), 97.43$ (2 C-3), 101.91 (2 C-5), 128.53 (2 C-1), 131.69 (2 C-6), 154.91 (2 C-4), 157.30 (2 C-2). EI/MS, m/z (I\%): [458 (30.58), 460 (60.44), 462 (31.30)] $\mathbf{M}^{+\bullet}$; [443 (51.59), 445 (100.00), 447 (51.06)] a; [229 (60.76), 231 (57.56)] b.

1-(2-Methoxyphenyl)-1-(3,4-dimethoxyphenyl)-2-methylpropane (20). ${ }^{13} \mathrm{C}$ NMR: 21.55 $\left(\mathrm{CHCH}_{3}\right), 21.96\left(\mathrm{CHCH}_{3}\right), 31.37\left[\mathrm{CH}\left(\mathrm{CH}_{3}\right)_{2}\right], 51.13\left[\mathrm{CHCH}\left(\mathrm{CH}_{3}\right)_{2}\right], 55.26\left(\mathrm{OCH}_{3}\right), 55.62$ $\left(\mathrm{OCH}_{3}\right), 55.68\left(\mathrm{OCH}_{3}\right), 110.78(\mathrm{C}-3), 110.99\left(\mathrm{C}-5{ }^{\prime}\right), 111.97\left(\mathrm{C}-2^{\prime}\right), 120.24\left(\mathrm{C}-6{ }^{\prime}\right), 120.56(\mathrm{C}-5)$, 126.65 (C-4), 127.37 (C-6), 133.42 (C-1), 137.65 (C-1'), 146.97 (C-4'), 148.49 (C-3'), 157.01 (C2). EI/MS, $m / z$ (I\%): $300(20.48) \mathbf{M}^{+\bullet} ; 257$ (78.57) a; 151 (100.00) b.

1,1-Bis(2,4-dimethoxyphenyl)-2-methylpropane (21). ${ }^{13} \mathrm{C} \quad \mathrm{NMR}: 21.61 \quad\left(\mathrm{CHCH}_{3}\right), 31.08$ $\left[\mathrm{CH}\left(\mathrm{CH}_{3}\right)_{2}\right], 43.67\left[\mathrm{CHCH}\left(\mathrm{CH}_{3}\right)_{2}\right], 55.37\left(2 \mathrm{OCH}_{3}\right), 55.45\left(2 \mathrm{OCH}_{3}\right), 98.59(2 \mathrm{C}-3), 103.99$ (2 C-5), 126.00 (2 C-1), 129.04 (2 C-6), 158.47 (2 C-4), 158.50 (2 C-2). EI/MS, m/z (I\%): 330 (13.61) $\mathbf{M}^{+\bullet} ; 287$ (100.00) a; 151 (56.90) b.

1-(2,4-Dimethoxyphenyl)-1-(3,4-dimethoxyphenyl)-2-methylpropane (22). ${ }^{13} \mathrm{C}$ NMR: 21.58 $\left(\mathrm{CHCH}_{3}\right), 22.03\left(\mathrm{CHCH}_{3}\right), 31.47\left[\mathrm{CH}\left(\mathrm{CH}_{3}\right)_{2}\right], 50.82\left[\mathrm{CHCH}\left(\mathrm{CH}_{3}\right)_{2}\right], 55.00\left(\mathrm{OCH}_{3}\right), 55.28$ $\left(\mathrm{OCH}_{3}\right), 55.66\left(\mathrm{OCH}_{3}\right), 55.70\left(\mathrm{OCH}_{3}\right), 98.55(\mathrm{C}-3), 104.38(\mathrm{C}-5), 111.09\left(\mathrm{C}-5^{\prime}\right), 111.89\left(\mathrm{C}-2^{\prime}\right)$, 120.13 (C-6'), 126.00 (C-1), 127.82 (C-6), 138.11 (C-1'), 146.91 (C-4'), 148.51 (C-3'), 157.95 (C-2), 158.73 (C-4). EI/MS, m/z (I\%): 330 (8.80) $\mathbf{M}^{+} ; 287$ (100.00) a; 151 (55.05) b.

1,1-Bis(2,5-dimethoxyphenyl)-2-methylpropane (23). ${ }^{13} \mathrm{C} \mathrm{NMR}: 21.55\left[\mathrm{CH}\left(\mathrm{CH}_{3}\right)_{2}\right], 31.12$ $\left[\mathrm{CH}\left(\mathrm{CH}_{3}\right)_{2}\right], 45.28\left[\mathrm{CHCH}\left(\mathrm{CH}_{3}\right)_{2}\right], 55.59\left(2 \mathrm{OCH}_{3}\right), 56.47\left(2 \mathrm{OCH}_{3}\right), 110.57(2 \mathrm{C}-4), 112.26(2$ C-3), 115.79 (2 C-6), 134.58 (2 C-1), 152.31 (2 C-2) 153.67 (2 C-5). EI/MS, m/z (I\%): 330 (32.18) $\mathbf{M}^{+\cdot} ; 287(66.28) \mathbf{a} ; 151(100.00) \mathbf{b}$.

1-(2,5-Dimethoxyphenyl)-1-(3,4-dimethoxyphenyl)-2-methylpropane (24). ${ }^{13} \mathrm{C}$ NMR: 21.48 $\left(\mathrm{CHCH}_{3}\right), 21.63\left(\mathrm{CHCH}_{3}\right), 31.40\left[\mathrm{CH}\left(\mathrm{CH}_{3}\right)_{2}\right], 51.23\left[\mathrm{CHCH}\left(\mathrm{CH}_{3}\right)_{2}\right], 55.54\left(\mathrm{OCH}_{3}\right), 55.73$ $\left(\mathrm{OCH}_{3}\right), 55.76\left(\mathrm{OCH}_{3}\right), 56.23\left(\mathrm{OCH}_{3}\right), 110.07(\mathrm{C}-4), 110.95(\mathrm{C}-5), 111.90\left(\mathrm{C}-3\right.$ and $\left.\mathrm{C}-2^{\prime}\right)$, 114.46 (C-6), 120.14 (C-6'), 135.02 (C-1), 137.43 (C-1'), 146.97 (C-4'), 148.45 (C-3'), 151.53 (C-2), 153.65 (C-5). EI/MS, m/z (I\%): 330 (40.82) $\mathbf{M}^{+\bullet} ; 287$ (100.00) a; 151 (94.90) b.

1-(2,3-Dimethoxyphenyl)-1-(3,4-dimethoxyphenyl)-2-methylpropane (25). ${ }^{13} \mathrm{C}$ NMR: 21.82 $\left(\mathrm{CHCH}_{3}\right), 21.88\left(\mathrm{CHCH}_{3}\right), 31.77\left[\mathrm{CH}\left(\mathrm{CH}_{3}\right)_{2}\right], 51.20\left[\mathrm{CHCH}\left(\mathrm{CH}_{3}\right)_{2}\right], 55.52\left(\mathrm{OCH}_{3}\right), 55.79$ (2 $\left.\mathrm{OCH}_{3}\right), 60.57\left(\mathrm{OCH}_{3}\right.$ at C-2), $109.56(\mathrm{C}-4), 111.00$ (C-5'), 111.86 (C-2'), 119.24 (C-6), 120.12 (C-6’), 123.90 (C-5), 137.67 (C-1'), 138.95 (C-1), 146.85 (C-2), 147.02 (C-4'), 148.53 (C-3'), 152.73 (C-3). EI/MS, m/z (I\%): 330 (36.73) $\mathbf{M}^{+\bullet} ; 287$ (52.19) a; 151 (100.00) b. 
1-(2,4,5-Trimethoxyphenyl)-1-(3,4-dimethoxyphenyl)-2-methylpropane $\quad(27) .{ }^{13} \mathrm{C} \quad \mathrm{NMR}$ : $21.14\left(\mathrm{CHCH}_{3}\right), 21.53\left(\mathrm{CHCH}_{3}\right), 31.09\left[\mathrm{CH}\left(\mathrm{CH}_{3}\right)_{2}\right], 50.47\left[\mathrm{CHCH}\left(\mathrm{CH}_{3}\right)_{2}\right], 55.33\left(\mathrm{OCH}_{3}\right), 55.36$ $\left(\mathrm{OCH}_{3}\right), 55.58\left(\mathrm{OCH}_{3}\right), 56.24\left(\mathrm{OCH}_{3}\right), 56.51\left(\mathrm{OCH}_{3}\right), 97.87(\mathrm{C}-3), 110.69\left(\mathrm{C}-5^{\prime}\right), 111.44\left(\mathrm{C}-2^{\prime}\right)$, 111.87 (C-6), 119.61 (C-6'), 125.00 (C-1), 137.52 (C-1'), 142.84 (C-5), 146.60 (C-4), 147.28 (C4'), 148.16 (C-3'), 151.07 (C-2'). EI/MS, m/z (I\%): 360 (30.61) $\mathbf{M}^{+} ; 317$ (100.00) a; 151 (55.80) b.

1,1-bis(4-Bromo-2,5-dimethoxyphenyl)-2-methylpropane (30). ${ }^{13} \mathrm{C} \mathrm{NMR:} 21.57 \mathrm{CH}\left(\mathrm{CH}_{3}\right)_{2}$, $30.26\left[\mathrm{CH}\left(\mathrm{CH}_{3}\right)_{2}\right], 47.06\left[\mathrm{CHCH}\left(\mathrm{CH}_{3}\right)_{3}\right], 56.36\left(2 \mathrm{OCH}_{3}\right), 57.15\left(2 \mathrm{OCH}_{3}\right), 109.11(2 \mathrm{C}-4)$, 114.34 (2 C-6), 116.59 (2 C-3), 132.75 (2 C-1), 150.14 (2 C-5), 152.42 (2 C-2). EI/MS, m/z (I\%): [486 (15.48), 488 (30.25), 490 (15.17)] $\mathbf{M}^{+\bullet}$; [443 (53.10), 445 (100.00), 447 (54.21)] a; [229 (94.70), $231(92.81)]$ b.

1,1-bis(5-Bromo-2,4-dimethoxyphenyl)-2-methylpropane (31). ${ }^{13} \mathrm{C} \mathrm{NMR:} 21.48\left(\mathrm{CHCH}_{3}\right)_{2}$, $30.13\left[\mathrm{CH}\left(\mathrm{CH}_{3}\right)_{2}\right], 45.12\left[\mathrm{CHCH}\left(\mathrm{CH}_{3}\right)_{2}\right], 55.87\left(2 \mathrm{OCH}_{3}\right), 56.29\left(2 \mathrm{OCH}_{3}\right), 97.23(2 \mathrm{C}-3)$, 101.79 (2 C-5), 126.65 (2 C-1) 132.90 (2 C-6), 154.53 (2 C-4), 157.76 (2 C-2). EI/MS, m/z (I\%): [486 (39.35), 488 (74.10), 490 (39.02)] $\mathbf{M}^{+}$; [443 (55.07), 445 (100.00), 447 (54.36)] a; [229 (60.37), 231 (58.09)] b.

1,1-Bis(3,4-dibromo-2,5-dimethoxyphenyl)-2-methylpropane $\quad$ (32). ${ }^{13} \mathrm{C} \quad \mathrm{NMR}: \quad 21.59$ $\left(\mathrm{CHCH}_{3}\right)_{2}, 32.78\left[\mathrm{CH}\left(\mathrm{CH}_{3}\right)_{2}\right], 46.44\left[\mathrm{CHCH}\left(\mathrm{CH}_{3}\right)_{2}\right], 57.03\left[2\left(\mathrm{OCH}_{3}\right.\right.$ at $\left.\left.\mathrm{C}-5\right)\right], 61.12\left[2\left(\mathrm{OCH}_{3}\right.\right.$ at C-2)], 110.89 (2 C-6), 114.21 (2 C-4), 122.48 (2 C-3) 137.03 (2 C-1), 150.87 (2 C-2), 153.65 (2 C-5). EI/MS, m/z (I\%): [642 (5.41), 644 (22.04), 646 (33.00), 648 (21.65), 650 (5.38)] M ${ }^{+\bullet}$; [599 (9.88), 601 (34.07), 603 (49.25), 605 (33.65), 607 (9.21)] a; [307 (52.04), 309 (100.00), 311 $(50.65)]$ b.

1-(3,5-Dibromo-2,4-dimethoxyphenyl)-1-(2,3-dibromo-4,5-dimethoxyphenyl)-2methylpropane (33). ${ }^{13} \mathrm{C}$ NMR: $20.21\left(\mathrm{CHCH}_{3}\right), 21.78\left(\mathrm{CHCH}_{3}\right), 33.04\left[\mathrm{CH}\left(\mathrm{CH}_{3}\right)_{2}\right], 51.71$ $\left[\mathrm{CHCH}\left(\mathrm{CH}_{3}\right)_{2}\right], 56.04\left(\mathrm{OCH}_{3}\right.$ at C-5'), $60.36\left(\mathrm{OCH}_{3}\right), 60.54\left(\mathrm{OCH}_{3}\right), 60.99\left(\mathrm{OCH}_{3}\right), 111.75(\mathrm{C}-$ 6'), 112.42 (C-5), 114.68 (C-3), 118.99 (C-2'), 121.83 (C-3'), 130.41 (C-6), 135.36 (C-1), 140.06 (C-1'), 145.97 (C-4'), 152.40 (C-5'), 153.52 (C-4), 156.67 (C-2). EI/MS, m/z (I\%): [642 (6.08), 644 (22.36), 646 (32.00), 648 (21.64), 650 (6.10)] $\mathbf{M}^{+\bullet}$; [599 (8.27), 601 (33.84), 603 (52.04), 605 (33.30), 607 (7.81)] a; [307 (52.67), 309 (100.00), 311 (50.84)] b.

1,1-Bis(2,5-dimethoxy-4-nitrophenyl)-2-methylpropane (36). ${ }^{13} \mathrm{C} \mathrm{NMR}: 21.43\left[\mathrm{CH}\left(\mathrm{CH}_{3}\right)_{2}\right]$, $30.01\left[\mathrm{CH}\left(\mathrm{CH}_{3}\right)_{2}\right], 48.30\left[\mathrm{CHCH}\left(\mathrm{CH}_{3}\right)_{2}\right], 56.34\left(2 \mathrm{OCH}_{3}\right) 57.58\left(2 \mathrm{OCH}_{3}\right), 108.30(2 \mathrm{C}-3)$, 116.54 (2 C-6), 138.10 (2 C-4) 138.81 (2 C-1), 147.60 (2 C-5), 151.45 (2 C-2) .EI/MS, m/z (I\%): $420(36.08) \mathbf{M}^{+\cdot} ; 377(38.21) \mathbf{a} ; 196(100.00) \mathbf{b}$.

1,1-Bis(2,4-dimethoxy-5-nitrophenyl)-2-methylpropane (37). ${ }^{13} \mathrm{C} \mathrm{NMR:} 21.62\left[\mathrm{CH}\left(\mathrm{CH}_{3}\right)_{2}\right]$, $28.94\left[\mathrm{CH}\left(\mathrm{CH}_{3}\right)_{2}\right], 47.22\left[\mathrm{CHCH}\left(\mathrm{CH}_{3}\right)_{2}\right], 56.00\left(2 \mathrm{OCH}_{3}\right) 56.64\left(2 \mathrm{OCH}_{3}\right), 96.33(2 \mathrm{C}-3)$, 123.96 (2 C-1), 127.87 (2 C-6) 132.06 (2 C-5), 154.39 (2 C-4), 162.68 (2 C-2). EI/MS, m/z (I\%): $420(6.35) \mathbf{M}^{+\bullet} ; 377(100.00) \mathbf{a} ; 196(50.20) \mathbf{b}$.

1,1,1-Trichloro-2-(2-methoxyphenyl)-2-(4-methoxyphenyl)ethane (38). EI/MS, m/z (I\%): [344 (13.96), 346 (13.27), 348 (4.50), 350 (0.56)] $\mathbf{M}^{+\bullet} ; 227$ (77.16) a; 121 (100.00) b. 
1,1,1-Trichloro-2,2-bis(2,5-dimethoxyphenyl)ethane (39). EI/MS, m/z (I\%): [404 (22.17), 406 (21.37), 408 (7.18), 410 (0.85)] $\mathbf{M}^{+\bullet} ; 287$ (79.73) a; 151 (100.00) b.

1,1,1-Trichloro-2,2-bis(2,4-dimethoxyphenyl)ethane (40). EI/MS, m/z (I\%): [404 (5.26), 406 (5.07), 408 (1.71), $410(0.20)] \mathbf{M}^{+\bullet} ; 287$ (100.00) a; 151 (40.54) b.

1,1,1-Trichloro-2,2-bis(2-methoxy-5-methylphenyl)ethane (41). EI/MS, m/z (I\%): $[372$ (15.90), 374 (15.33), 376 (5.08), 378 (0.60)] $\mathbf{M}^{+} ; 255$ (92.00) a; 135 (100.00) b.

1,1,1-Trichloro-2,2-bis(2,4,5-trimethoxyphenyl)ethane (42). EI/MS, m/z (I\%): [464 (9.43), 466 (9.21), 468 (3.14), 470 (0.39)] $\mathbf{M}^{+\bullet} ; 347$ (100.00) a; 181 (27.52) b.

Compounds $2,3,5,7,12,14,15,17,26,29$, and 35 were synthesized by the following general procedure.

A solution of the appropriate alcohol $(10 \mathrm{mmol})$ in glacial acetic acid $(25 \mathrm{ml})$ was added to a stirred solution of appropriate aromatic substrate $(50 \mathrm{mmol})$ in glacial acetic acid $(25 \mathrm{ml}) / 70 \%$ $\mathrm{H}_{2} \mathrm{SO}_{4}(25 \mathrm{ml})$ at $20{ }^{\circ} \mathrm{C}$. After standing at room temperature overnight, the mixture was poured onto crushed ice and the oil obtained was extracted with ethyl acetate, neutralized and concentrated under reduced pressure. The unreacted aromatic substrate was removed by steam distillation. Finally the residue was extracted with ethyl acetate, dried and concentrated at reduced pressure. The utilized alcohol and aromatic substrate, together with the purification method, are reported below.

For compounds 2, 3, 5, and 7, commercial sec-phenylethanol was utilized and aromatic substrates were 4-methylanisole, 1,4-dimethoxybenzene, 4-bromoanisole, and 1,3dimethoxybenzene, respectively. The crude products were chromatographed over silica gel, employing cyclohexane:diethyl ether (95:5) as eluent.

For compounds 12,14, and 15, the alcohol utilized was 1-(2-methoxyphenyl)ethanol, ${ }^{14}$ and the aromatic substrates were anisole, 3-chloroanisole, and 3-bromoanisole, respectively. The crude products were chromatographed over silica gel, employing cyclohexane:diethyl ether (80:20) for 12 and cyclohexane:diethyl ether (95:5) for $\mathbf{1 4}$ and 15, as eluent.

For compound 17, commercial 4-methoxy- $\alpha$-methylbenzyl alcohol and 1,3dimethoxybenzene were utilized. The crude product was chromatographed over silica gel, employing cyclohexane:ethyl acetate (96:4) as eluent.

For compounds 26, 29, and 35, the alcohol used was 1-(3,4-dimethoxyphenyl)-2methylpropan-1-ol, ${ }^{15}$ and the aromatic substrates were 1,2,3-trimethoxybenzene, 4-methylanisole and 5-chloro-1,3-dimethoxybenzene, respectively. The crude products were chromatographed over silica gel, employing as eluent cyclohexane:diethyl ether (90:10) for 26 and 29, and cyclohexane:ethyl acetate (90:10) for 35 .

1-(5-Methyl-2-methoxyphenyl)-1-phenylethane (2). Pale yellow oil (yield 55\%). ${ }^{13} \mathrm{C}$ NMR: $20.68\left(\mathrm{CH}_{3}\right), 20.88\left(\mathrm{CH}_{3}\right), 37.25\left(\mathrm{CHCH}_{3}\right), 55.53\left(\mathrm{OCH}_{3}\right), 110.61(\mathrm{C}-3), 125.59(\mathrm{C}-4), 127.25$ (C-4), 127.65 (C-2',6'), 128.03 (C-3',5'), 128.34 (C-6), 129.52 (C-5), 134.61 (C-1), 146.41 (C-1'), 154.72 (C-2). EI/MS, m/z (I\%): 226 (66.88) $\mathbf{M}^{+} ; 211$ (73.97) a; 91 (100.00) b. 
1-(2,5-Dimethoxyphenyl)-1-phenylethane (3). Yellow oil (yield 85\%). ${ }^{13} \mathrm{C}$ NMR: 20.79 $\left(\mathrm{CHCH}_{3}\right), 37.45\left(\mathrm{CHCH}_{3}\right), 55.36\left(\mathrm{OCH}_{3}\right), 55.91\left(\mathrm{OCH}_{3}\right), 110.30(\mathrm{C}-4), 111.41(\mathrm{C}-3), 114.62(\mathrm{C}-$ 6), 125.66 (C-4'), 127.56 (C-2',6'), 128.03 (C-3',5'), 136.16 (C-1), 145.97 (C-1'), 151.08 (C-2), 153.49 (C-5). EI/MS, m/z (I\%): 242 (100.00) $\mathbf{M}^{+\bullet} ; 227$ (36.22) a; 91 (61.46) b.

1-(5-Bromo-2-methoxyphenyl)-1-phenylethane (5). White oil (yield 40\%). ${ }^{13} \mathrm{C}$ NMR: 20.74 $\left(\mathrm{CHCH}_{3}\right), 37.38\left(\mathrm{CHCH}_{3}\right), 55.61\left(\mathrm{OCH}_{3}\right), 112.27(\mathrm{C}-3), 112.94(\mathrm{C}-5), 125.94(\mathrm{C}-4), 127.59(\mathrm{C}-$ 2',6'), 128.20 (C-3',5'), 129.69 (C-4), 130.47 (C-6), 137.19 (C-1), 145.44 (C-1'), 155.90 (C-2). EI/MS, m/z (I\%): [290 (48.67), 292 (48.31)] $\mathbf{M}^{+\bullet}$; [275 (31.41), 277 (31.06)] a; 91 (100.00) b.

1-(2,4-Dimethoxyphenyl)-1-phenylethane (7). White oil (yield 90\%). ${ }^{13} \mathrm{C}$ NMR: 21.04 $\left(\mathrm{CHCH}_{3}\right), 36.93\left(\mathrm{CHCH}_{3}\right) 55.26\left(\mathrm{OCH}_{3}\right), 55.38\left(\mathrm{OCH}_{3}\right), 98.60(\mathrm{C}-3), 103.88(\mathrm{C}-5), 125.55(\mathrm{C}-$ 4'), 127.48 (C-1), 127.48 (C-2',6'), 127.93 (C-6), 128.03 (C-3',5'), 146.72 (C-1'), 157.72 (C-2), 159.03 (C-4). EI/MS, m/z (I\%): 242 (55.11) $\mathbf{M}^{+\bullet} ; 227$ (100.00) a; 91 (51.07) b.

1-(2-Methoxyphenyl)-1-(4-methoxyphenyl)ethane (12). Yellow oil (yield $70 \%)$. ${ }^{13} \mathrm{C}$ NMR: $21.01\left(\mathrm{CHCH}_{3}\right), 36.48\left(\mathrm{CHCH}_{3}\right), 55.14\left(\mathrm{OCH}_{3}\right), 55.39\left(\mathrm{OCH}_{3}\right), 110.51(\mathrm{C}-3), 113.43\left(\mathrm{C}-3{ }^{\prime}, 5^{\prime}\right)$, 120.45 (C-5), 126.91 (C-4), 127.49 (C-6), 128.56 (C-2',6'), 135.22 (C-1), 138.45 (C-1'), 156.71 (C-2), 157.55 (C-4'). EI/MS, m/z (I\%): 242 (47.20) $\mathbf{M}^{+} ; 227$ (76.33) a; 121 (100.00) b.

1-(2-Methoxyphenyl)-1-(2-chloro-4-methoxyphenyl)ethane (14). White oil (yield $72 \%) .{ }^{13} \mathrm{C}$ NMR: $20.08\left(\mathrm{CHCH}_{3}\right), 34.10\left(\mathrm{CHCH}_{3}\right), 55.33\left(\mathrm{OCH}_{3}\right), 55.44\left(\mathrm{OCH}_{3}\right), 110.53(\mathrm{C}-3), 112.57(\mathrm{C}-$ 5'), 114.60 (C-3'), 120.27 (C-5), 127.14 (C-4), 127.27 (C-6), 128.72 (C-6'), 133.90 (C-1), 134.28 (C-2'), 135.79 (C-1'), 156.97 (C-2), 157.99 (C-4'). EI/MS, m/z (I\%): [276 (41.97), 278 (13.68)] $\mathbf{M}^{+} ;$[261 (52.14), 263 (16.73)] a; [155 (100.00), 157 (31.49)] b.

1-(2-Methoxyphenyl)-1-(2-bromo-4-methoxyphenyl)ethane (15). White oil (yield $60 \%) .{ }^{13} \mathrm{C}$ NMR: $20.24\left(\mathrm{CHCH}_{3}\right), 36.70\left(\mathrm{CHCH}_{3}\right), 55.35\left(\mathrm{OCH}_{3}\right), 55.44\left(\mathrm{OCH}_{3}\right), 110.51(\mathrm{C}-3), 113.24(\mathrm{C}-$ 5'), 117.80 (C-3'), 120.25 (C-5), 124.87 (C-2'), 127.16 ( C-4), 127.18 (C-6), 128.68 (C-6'), 133.92 (C-1), 137.45 (C-1'), 157.07 (C-2), 157.98 (C-4). EI/MS, m/z (I\%): [320 (47.76), 322 (47.63)] $\mathrm{M}^{\circ}$; [305 (46.08), 307 (45.33)] a; [199 (100.00), 201 (97.14)] b.

1-(2,4-Dimethoxyphenyl)-1-(4-methoxyphenyl)ethane (17). Yellow oil (yield 50\%). ${ }^{13} \mathrm{C}$ NMR: $21.19\left(\mathrm{CHCH}_{3}\right), 36.14\left(\mathrm{CHCH}_{3}\right), 55.19\left(\mathrm{OCH}_{3}\right), 55.28\left(\mathrm{OCH}_{3}\right), 55.43\left(\mathrm{OCH}_{3}\right), 98.68(\mathrm{C}-3)$, 104.00 (C-5), 113.47 (C-3',5'), 127.86 (C-6), 128.02 (C-1), 128.47 (C-2',6'), 138.84 (C-1'), 157.55 (C-4'), 157.71 (C-2), 159.01 (C-4). EI/MS, m/z (I\%): 272 (38.27) $\mathrm{M}^{+\bullet} ; 257$ (100.00) a; $121(61.58) \mathbf{b}$.

1-(2,3,4-Trimethoxyphenyl)-1-(3,4-dimethoxyphenyl)-2-methylpropane (26). Pale yellow oil (yield 85\%). ${ }^{13} \mathrm{C}$ NMR: $21.86\left(\mathrm{CHCH}_{3}\right), 21.96\left(\mathrm{CHCH}_{3}\right), 31.76\left[\mathrm{CH}\left(\mathrm{CH}_{3}\right)_{2}\right], 51.30$ $\left[\mathrm{CHCH}\left(\mathrm{CH}_{3}\right)_{2}\right], 55.71\left(\mathrm{OCH}_{3}\right), 55.77\left(2 \mathrm{OCH}_{3}\right), 60.50\left(\mathrm{OCH}_{3}\right), 60.74\left(\mathrm{OCH}_{3}\right), 107.42(\mathrm{C}-5)$, 111.08 (C-5'), 111.82 (C-2'), 120.15 (C-6'), 121.51 (C-6), 131.28 (C-1), 138.01 (C-1'), 142.21 (C-3), 147.04 (C-4'), 148.59 (C-3'), 151.57 (C-4), 151.76 (C-2). EI/MS, m/z (I\%): 360 (21.77) $\mathrm{M}^{+\cdot} ; 317(100.00) \mathbf{a} ; 151(73.95) \mathbf{b}$.

1-(5-Methyl-2-methoxyphenyl)-1-(3,4-dimethoxyphenyl)-2-methylpropane (29). White crystals from EtOH (yield 60\%), m.p. $71{ }^{\circ} \mathrm{C} .{ }^{13} \mathrm{C}$ NMR: $20.58\left(\mathrm{CH}_{3}\right), 21.39\left(\mathrm{CHCH}_{3}\right), 21.78$ 
$\left(\mathrm{CHCH}_{3}\right), 31.15\left[\mathrm{CH}\left(\mathrm{CH}_{3}\right)_{2}\right], 51.20\left[\mathrm{CHCH}\left(\mathrm{CH}_{3}\right)_{2}\right], 55.43\left(\mathrm{OCH}_{3}\right), 55.55\left(\mathrm{OCH}_{3}\right), 55.60$ $\left(\mathrm{OCH}_{3}\right), 110.80$ and 110.95 (C-3 and C-5'), 112.03 (C-2'), 120.04 (C-6'), 126.77 (C4), 128.07 (C6), 129.37 (C-5) 133.14 (C-1), 137.68 (C-1'), 146.82 (C-4'), 148.34 (C-3'), 154.85 (C-2). EI/MS, $\mathrm{m} / \mathrm{z}(\mathrm{I} \%): 314$ (20.41) $\mathrm{M}^{+} ; 271$ (100.00) a; 151 (83.37) b.

1-(6-Chloro-2,4-dimethoxyphenyl)-1-(3,4-dimethoxyphenyl)-2-methylpropane (35). White crystals from EtOH (yield 65\%), m.p. $85{ }^{\circ} \mathrm{C} .{ }^{13} \mathrm{C}$ NMR: $21.49(\mathrm{CHCH} 3), 22.49\left(\mathrm{CHCH}_{3}\right), 28.72$ $\left[\mathrm{CH}\left(\mathrm{CH}_{3}\right)_{2}\right], 53.67\left[\mathrm{CHCH}\left(\mathrm{CH}_{3}\right)_{2}\right], 55.30\left(2 \mathrm{OCH}_{3}\right), 55.71\left(2 \mathrm{OCH}_{3}\right), 98.47(\mathrm{C}-3), 106.11(\mathrm{C}-5)$, 110.61 (C-5'), 112.40 (C-2'), 121.15 (C-6'), 123.96 (C-1), 135.18 (C-6), 136.36 (C-1'), 147.04 (C-4'), 148.21 (C-3'), 158.51 (C-2), 159.25 (C-4). EI/MS, m/z (I\%): [364 (27.10), 366 (9.31)] $\mathrm{M}^{+} ;$[321 (100.00), 323 (36.28)] a; 151 (70.20) b.

1-(4-Methyl-2-methoxyphenyl)-1-phenylethane (6). To a solution of 3-methylanisole (13 mmol), $N, N, N^{\prime}, N$ '-tetramethyl-ethylenediamine (TMEDA) (13 mmol) in anhydrous diethyl ether $(10 \mathrm{ml}), 1.6 \mathrm{M} \mathrm{n}$-BuLi solution in hexane $(16 \mathrm{mmol})$ was added at $0{ }^{\circ} \mathrm{C}$, under an argon atmosphere. The mixture was then allowed to stir at room temperature for $2 \mathrm{~h}$, then a solution of commercial (1-bromoethyl)benzene $(13 \mathrm{mmol})$ in anhydrous diethyl ether $(10 \mathrm{ml})$ was added. After stirring overnight at room temperature, the reaction was quenched with solution of ammonium chloride, then extracted with diethyl ether, neutralized and dried. After solvent evaporation in vacuo, the oily residue was chromatographed over silica gel. Elution with cyclohexane gave pure 6 (yield $65 \%$ ) as a white oil. ${ }^{13} \mathrm{C} \mathrm{NMR}: 20.92\left(\mathrm{CHCH}_{3}\right), 21.38\left(\mathrm{CH}_{3}\right)$, $37.09\left(\mathrm{CHCH}_{3}\right), 55.36\left(\mathrm{OCH}_{3}\right), 111.57(\mathrm{C}-3), 121.02(\mathrm{C}-5), 125.57(\mathrm{C}-4), 127.42(\mathrm{C}-6), 127.64$ (C-2',6'), 128.04 (C-3',5'), 131.94 (C-1), 136.85 (C-4), 146.57 (C-1'), 156.65 (C-2). EI/MS, m/z (I\%): 226 (56.93) $\mathbf{M}^{+\bullet} ; 211$ (80.34) a; 91 (100.00) b.

1,1-bis-(5-Methyl-2-methoxyphenyl)-2-methylpropane (28). To a solution of 4-methylanisole $(81 \mathrm{mmol})$ and isobutyraldehyde $(27 \mathrm{mmol})$ in glacial acetic acid $(50 \mathrm{ml}), 98 \% \mathrm{H}_{2} \mathrm{SO}_{4}(30 \mathrm{ml})$ was added dropwise with stirring. External cooling with ice was necessary in order to maintain the reaction temperature below $20^{\circ} \mathrm{C}$. After standing at room temperature overnight, the mixture was poured onto crushed ice and the oil obtained was extracted with ethyl acetate, neutralized, and concentrated under reduced pressure. The unreacted 4-methylanisole was removed by steam distillation. Finally, the residue was extracted with ethyl acetate, dried and concentrated at reduced pressure. Pure $\mathbf{2 8}$ was obtained by chromatography over silica gel, employing cyclohexane: diethyl ether (90:10), as eluent. White crystals from $\mathrm{EtOH}$ (yield 60\%), m.p. $101{ }^{\circ} \mathrm{C} .{ }^{13} \mathrm{C}$ NMR: $20.78\left(2 \mathrm{CH}_{3}\right), 21.58\left[\mathrm{CH}\left(\mathrm{CH}_{3}\right)_{2}\right], 31.09\left[\mathrm{CH}\left(\mathrm{CH}_{3}\right)_{2}\right], 44.45\left[\mathrm{CHCH}\left(\mathrm{CH}_{3}\right)_{2}\right]$, $55.84\left(2 \mathrm{OCH}_{3}\right), 111.13$ (2 C-3), 126.72 ( $\left.2 \mathrm{C}-4\right), 129.23$ ( $\left.2 \mathrm{C}-5\right), 129.58$ (2 C-6), 133.11 (2 C1), 155.70 (2 C-2). EI/MS, m/z (I\%): 298 (18.37) $\mathbf{M}^{+\cdot} ; 255$ (71.20) a; 135 (100.00) b.

Compounds 4, 11 and 44 were obtained by the following nitration procedure. A solution of 1 or 3 or $39(2.4 \mathrm{mmol})$ in $\mathrm{Ac}_{2} \mathrm{O}(25 \mathrm{ml})$ was placed in a two-necked round-bottomed flask with a mechanical stirrer and a reflux condenser protected by a $\mathrm{CaCl}_{2}$ tube. To the stirred solution, $\mathrm{CuNO}_{3} \cdot 3 \mathrm{H}_{2} \mathrm{O}(1.2 \mathrm{mmol}$ for $\mathbf{4}$ and $\mathbf{1 1}$, and $2.4 \mathrm{mmol}$ for $\mathbf{4 4})$ was added. The mixture was heated at $40-50{ }^{\circ} \mathrm{C}$ during $0.5 \mathrm{~h}$. The cooled mixture was poured in water, then extracted with $\mathrm{CHCl}_{3}$, 
neutralized, dried, and evaporated in vacuo. The crude products were chromatographed over silica gel, employing cyclohexane: ethyl acetate (90:10) and (95:5) for $\mathbf{3}$ and $\mathbf{5}$, respectively, as eluent. Pure $\mathbf{4 4}$ was obtained by crystallization of the crude product.

1-(2-Methoxy-5-nitrophenyl)-1-phenylethane (4). White crystals from EtOH (yield 83\%), m.p. $101{ }^{\circ} \mathrm{C} .{ }^{13} \mathrm{C}$ NMR: $20.67\left(\mathrm{CHCH}_{3}\right), 37.49\left(\mathrm{CHCH}_{3}\right), 56.01\left(\mathrm{OCH}_{3}\right), 109.93(\mathrm{C}-3), 123.24(\mathrm{C}-6)$, 123.76 (C-4), 126.21 (C-4'), 127.44 (C-2',6'), 128.73 (C-3',5'), 135.86 (C-1), 242 (C-5), 144.63 (C-1'), 161.79 (C-2). EI/MS, m/z (I\%): 257 (42.93) $\mathbf{M}^{+\cdot} ; 242$ (55.07) a; 91 (100.00) b.

1-(2,5-Dimethoxy-4-nitrophenyl)-1-phenylethane (11). Yellow crystals from EtOH (yield $80 \%$ ), m.p. $89{ }^{\circ} \mathrm{C} .{ }^{13} \mathrm{C}$ NMR: $20.33\left(\mathrm{CHCH}_{3}\right), 37.89\left(\mathrm{CHCH}_{3}\right) 56.14(\mathrm{OCH} 3), 57.03(\mathrm{OCH} 3)$, 107.78 (C-3), 114.08 (C-6), 126.37 (C-4'), 127.56 (C-2',6'), 128.38 (C-3',5'), 136.98 (C-4), 143.06 (C-1), 144.27 (C-1'), 147.80 (C-5), 150.17 (C-2). EI/MS, m/z (I\%): 287 (73.58) $\mathbf{M}^{+\cdot} ; 272$ (12.45) a; $91(100.00) \mathbf{b}$.

1-(2,5-Dimethoxy-4-nitrophenyl)-1-(4-nitrophenyl)-ethane (13). This compound was obtained from $11(2.4 \mathrm{mmol})$ and $\mathrm{CuNO}_{3} \cdot 3 \mathrm{H}_{2} \mathrm{O}(1.2 \mathrm{mmol})$ by the nitration procedure describe above. The crude product was chromatographed over silica gel, employing cyclohexane: ethyl acetate (80:20) as eluent. Yellow crystals from EtOH (yield $70 \%$ ), m.p. $130{ }^{\circ} \mathrm{C} .{ }^{13} \mathrm{C}$ NMR: 20.13 $\left(\mathrm{CHCH}_{3}\right), 38.29\left(\mathrm{CHCH}_{3}\right) 56.12\left(\mathrm{OCH}_{3}\right), 57.24\left(\mathrm{OCH}_{3}\right), 107.94(\mathrm{C}-3), 113.85(\mathrm{C}-6), 123.67(\mathrm{C}-$ 3',5'), 128.30 (C-2',6'), 137.65 (C-4), 140.47 (C-1), 146.44 (C-4'), 147.75 (C-5), 150.15 (C-2), 152.25 (C-1'). EI/MS, $m / z$ (I\%): 332 (100.00) $\mathbf{M}^{+\bullet} ; 317$ (4.69) a; 136 (31.85) b.

1-(2,5-[Dimethoxy- $\left.\left.{ }^{2} \mathbf{H}_{6}\right]-4-n i t r o p h e n y l\right)-1-(4-n i t r o p h e n y l)-e t h a n e ~\left(13-d_{6}\right)$. This compound was synthesized by the same reaction series as for 13, using 1,4-hexadeuterodimethoxybenzene. Analytical, ${ }^{1} \mathrm{H}$ - and ${ }^{13} \mathrm{C}$ NMR data confirmed the assigned structure. EI/MS, $\mathrm{m} / \mathrm{z}$ (I\%): 338 (100.00) $\mathbf{M}^{+} ; 323$ (4.30) a; 138 (20.30) b.

1,1,1-Trichloro-2,2-bis(2,5-dimethoxy-4-nitrophenyl)-ethane (44). Yellow crystals from EtOH (yield 85\%), m.p. $187{ }^{\circ} \mathrm{C} .{ }^{13} \mathrm{C}$ NMR: 53.21 $\left(\mathrm{CHCCl}_{3}\right), 56.59\left(2 \mathrm{OCH}_{3}\right), 57.36\left(2 \mathrm{OCH}_{3}\right)$, $99.94\left(\mathrm{CCl}_{3}\right), 108.16$ (2 C-3), 116.21 (2 C-6), 132.53 (2 C-1), 138.86 (2 C-4), 146.70 (2 C-5), 151.24 (2 C-2). EI/MS, m/z (I\%): [494 (13.55), 496 (13.15), 498 (4.54), $500(0.56)] \mathrm{M}^{+} ; 377$ (73.31) a; $196(100.00)$ b.

1-(4-Amino-2,5-dimethoxyphenyl)-1-phenylethane (8). To a solution of $11(3.5 \mathrm{mmol})$ in EtOH $(25 \mathrm{ml})$ was added $10 \% \mathrm{Pd}-\mathrm{C}(0.35 \mathrm{mmol})$. The mixture was hydrogenated in a Parr apparatus for $72 \mathrm{~h}$. Elimination of catalyst and evaporation of solvent at reduced pressure produced a solid residue. Crystallization of the crude product from EtOH afforded pure $\mathbf{8}$ as pale pink crystals (yield $75 \%$ ), m.p. $88{ }^{\circ} \mathrm{C}$. IR (Nujol): vmax 3350 and $3450 \mathrm{~cm}^{-1}$ (NH2). ${ }^{13} \mathrm{C}$ NMR: $21.09\left(\mathrm{CHCH}_{3}\right), 36.94\left(\mathrm{CHCH}_{3}\right), 56.28\left(\mathrm{OCH}_{3}\right), 56.34\left(\mathrm{OCH}_{3}\right), 100.32(\mathrm{C}-3), 111.40(\mathrm{C}-6)$, 124.48 (C-1), 125.46 (C-4'), 127.48 (C-2',6'), 127.98 (C-3',5'), 134.79 (C-4), 141.31 (C-5), 146.97 (C-1'), 151.40 (C-2). EI/MS, m/z (I\%): 257 (65.32) $\mathbf{M}^{+\cdot} ; 242$ (100.00) a; 91 (24.61) b.

1-(4- $N$-Methyl-2,5-dimethoxyphenyl)-1-phenylethane (9) and 1-(4- $N, N$-Dimethylamino-2,5di-methoxyphenyl) -1-phenylethane (10). Methyl iodide $(30 \mathrm{mmol})$ and $\mathrm{K}_{2} \mathrm{CO}_{3}(30 \mathrm{mmol})$ were added to a solution of $\mathbf{8}(6 \mathrm{mmol})$ in acetone $(20 \mathrm{ml})$. The mixture was refluxed for $5 \mathrm{~h}$. 
After cooling, the solid material was removed by filtration and the solution was diluted with water and extracted with diethyl ether. The ethereal extracts were treated with $10 \%$ aqueous sodium hydroxide solution, then neutralized, dried and evaporated in vacuo. The residue obtained was chromatographed on silica gel employing cyclohexane:ethyl acetate (90:10) as eluent. The elution order of the compounds was: $\mathbf{1 0 , 9 .}$

Pure 9 was obtained as pale pink crystals from petroleum ether (b.p. $40-60{ }^{\circ} \mathrm{C}$ ) (yield $25 \%$ ), m.p. $53{ }^{\circ} \mathrm{C}$. IR (Nujol): vmax $3420 \mathrm{~cm}^{-1}(\mathrm{NH}) .{ }^{13} \mathrm{C}$ NMR: $21.07\left(\mathrm{CHCH}_{3}\right), 30.41\left(\mathrm{NCH}_{3}\right), 36.74$ $\left(\mathrm{CHCH}_{3}\right), 56.12\left(\mathrm{OCH}_{3}\right), 56.57\left(\mathrm{OCH}_{3}\right), 95.64(\mathrm{C}-3), 110.00(\mathrm{C}-6), 121.77(\mathrm{C}-1), 125.35\left(\mathrm{C}-4{ }^{\prime}\right)$, 127.45 (C-2',6'), 127.93 (C-3',5'), 138.31 (C-4), 141.01 (C-5), 147.13 (C-1'), 151.69 (C-2). EI/MS, m/z (I\%): $271(63.57) \mathbf{M}^{+\cdot} ; 256$ (100.00) a; 91 (12.14) b.

Pure 10 was obtained as pale pink crystals from petroleum ether (b.p. $40-60{ }^{\circ} \mathrm{C}$ ) (yield 50\%), m.p. $58{ }^{\circ} \mathrm{C} .{ }^{13} \mathrm{C}$ NMR: $20.84\left(\mathrm{CHCH}_{3}\right), 37.08\left(\mathrm{CHCH}_{3}\right), 43.19\left[\mathrm{~N}\left(\mathrm{CH}_{3}\right)_{2}\right], 55.79\left(\mathrm{OCH}_{3}\right), 56.40$ $\left(\mathrm{OCH}_{3}\right), 103.27$ (C-3), 111.63 (C-6), 125.51 (C-1), 125.51 (C-4'), 127.47 (C-2',6'), 127.96 (C3',5'), 140.90 (C-5), 146.33 (C-4), 146.49 (C-1'), 150.86 (C-2). EI/MS, m/z (I\%): 285 (79.78) $\mathbf{M}^{+\cdot} ; 270(100.00) \mathbf{a} ; 91(20.40) \mathbf{b}$.

1,1-bis-(2-Methoxyphenyl)-ethane (16). To a solution of 1,1-bis-(2-methoxyphenyl)-ethanol ${ }^{16}$ $(8 \mathrm{mmol})$ in glacial acetic acid $(25 \mathrm{ml})$ was added $10 \% \mathrm{Pd}-\mathrm{C}(0.8 \mathrm{mmol})$. The mixture was hydrogenated in a Parr apparatus for $24 \mathrm{~h}$. Elimination of catalyst and evaporation of solvent at reduced pressure produced a solid residue. Crystallization of the crude product from $\mathrm{EtOH}$ afforded pure 16 as white crystals (yield 90\%), m.p. $68{ }^{\circ} \mathrm{C} .{ }^{13} \mathrm{C}$ NMR: $19.88\left(\mathrm{CHCH}_{3}\right), 31.28$ $\left(\mathrm{CHCH}_{3}\right), 55.57\left(2 \mathrm{OCH}_{3}\right), 110.64$ (2 C-3), 120.24 (2 C-5), 126.75 (2 C-4), 127.68 (2 C-6), 134.84 (2 C-1), 157.04 (2 C-2). EI/MS, m/z (I\%): 242 (58.48) $\mathbf{M}^{+\bullet} ; 227$ (48.86) a; 121 (100.00) b.

\section{1-(2,3-Dibromo-5,6-dimethoxyphenyl)-1-(2,3-dibromo-4,5-dimethoxyphenyl)-2-}

methylpropane (34). A solution of $25(5 \mathrm{mmol})$ in $\mathrm{CHCl}_{3}(10 \mathrm{ml})$ was stirred at room temperature during the dropwise addition of a solution of bromine $(50 \mathrm{mmol})$ in $\mathrm{CHCl}_{3}(20 \mathrm{ml})$. The solution was then set aside at room temperature until the hydrogen bromide gas evolution was complete ( 1 week). The solid obtained after evaporation of the solvent in vacuo, was crystallized from EtOH. White crystals (yield 92\%), m.p. $205{ }^{\circ} \mathrm{C} .{ }^{13} \mathrm{C} \mathrm{NMR}: 20.40\left(\mathrm{CHCH}_{3}\right)$, $22.15\left(\mathrm{CHCH}_{3}\right), 30.71\left[\mathrm{CH}\left(\mathrm{CH}_{3}\right)_{2}\right], 55.86$ and $56.15\left(2 \mathrm{OCH}_{3}\right.$ at $\mathrm{C}-5$ and $\left.\mathrm{C}-5{ }^{\prime}\right), 58.25$ $\left[\mathrm{CHCH}\left(\mathrm{CH}_{3}\right)_{2}\right], 59.64$ and $60.45\left(2 \mathrm{OCH}_{3}\right.$ at C-6 and C-4'), $114.98\left(\mathrm{C}-6{ }^{\prime}\right), 115.71(\mathrm{C}-4), 119.63$ (C-2'), 120.00 (C-3), 121.55 (C-3'), 121.63 (C-2), 137.20 (C-1), 139.29 (C-1'), 145.93 (C-4'), 147.14 (C-6), 151.41 (C-5'), 152.35 (C-5). EI/MS, m/z (I\%): [642 (4.52), 644 (17.43), 646 (25.34), 648 (17.18), 650 (4.56)] $\mathbf{M}^{+\bullet}$; [599 (7.69), 601 (29.66), 603 (44.50), 605 (29.21), 607 (7.39)] a; [307 (50.69), 309 (100.00), 311 (51.00)] b. 
1,1,1-Trichloro-2,2-bis-(4-bromo-2,5-dimethoxyphenyl)ethane (43). Compound 43 was obtained from $39^{11}$ by the bromination procedure describe above, using 2 equiv. of bromine (reaction time $48 \mathrm{~h}$ ). The obtained solid was crystallized from EtOH. White crystals (yield 90\%), m.p. $167{ }^{\circ} \mathrm{C} .{ }^{13} \mathrm{C}$ NMR: $52.60\left(\mathrm{CHCCl}_{3}\right), 56.72\left(2 \mathrm{OCH}_{3}\right), 57.06\left(2 \mathrm{OCH}_{3}\right), 101.44\left(\mathrm{CCl}_{3}\right)$, 111.57 (2 C-4), 114.16 (2 C-6), 116.83 (2 C-3), 126.81 (2 C-1), 149.69 (2 C-5), 152.28 (2 C-2). EI/MS, m/z (I\%): [560 (7.12), 562 (20.99), 564 (22.87), 566 (11.53), 568 (2.76), 570 (0.28)] $\mathbf{M}^{+\bullet}$; [443 (50.21), 445 (100.00), 447 (50.23)] a; [229 (68.97), 231 (66.52)] b.

\section{Acknowledgments}

Financial support from the 'Fondi Ricerca Scientifica' of the University of Palermo is gratefully acknowledged.

\section{References}

1. Ceraulo, L.; Natoli, M.C.; Agozzino, P.; Ferrugia, M.; Lamartina L. Org. Mass Spectrom. 1991, $26,857$.

2. Natoli, M.C.; Ceraulo, L.; Ferrugia, M.; Lamartina, L. Eur. Mass Spectrom. 1999, 5, 363.

3. Ceraulo, L.; Agozzino, P.; Ferrugia, M.; Lamartina, L. Natoli, M.C. Org. Mass Spectrom. 1992, 27, 1127.

4. Natoli, M.C.; Agozzino, P.; Ceraulo, L.; Ferrugia, M.; Lamartina, L. Eur. Mass Spectrom. 1995, $1,73$.

5. Natoli, M.C.; Ceraulo, L.; Ferrugia, M.; Lamartina, L. Eur. Mass Spectrom. 1997, 3, 361.

6. Buongiorno, D.; Ceraulo, L.; Lamartina, L.; Natoli, M.C. Rapid Commun. Mass Spectrom. 2000, 14, 203.

7. Kuck, D. Mass Spectrom. Rev. 1990, 9, 187 and references cited therein.

8. McLafferty, F.W.; Turecek, F. Interpretation of Mass Spectra; $4^{\text {th }}$ Edn; University Science Books: Mill Valley, 1993.

9. Levy, G. C.; Nelson, G. L. Carbon-13 Nuclear Magnetic Resonance for Organic Chemists; Wiley-Interscience: N.Y., 1972.

10. Olah, G. A.; Surya Prakash, G. K.; Sommer, J. Superacids, Wiley: New York, 1985.

11. Fontana, G.; Frenna, V.; Lamartina, L.; Natoli, M.C.; Noto, R. J. Phys. Org. Chem. 2002, 15, 108.

12. Natoli, M.C.; Agozzino, P.; Ceraulo, L., Lamartina, L. Gazz. Chim. Ital. 1983, 113, 493.

13. Natoli, M.C.; Ceraulo, L.; Lamartina, L. Gazz. Chim. Ital. 1988, 119, 45.

14. Marvel, C.S.; Hein, D.W. J. Am. Chem. Soc. 1948, 70, 895. 
15. Roberti, P.C.; York, R.F.; McGregor, W.S. J. Am. Chem. Soc. 1950, 72, 5760.

16. Coleman, G.H.; Holst, W.H.; Maxwell, R.D. J. Am. Chem. Soc. 1936, 58, 2310. 